\title{
Spreading Pressures of Water and $n$-Propanol on Polymer Surfaces
}

\author{
H. J. BUSSCHER,* G. A. M. KIP,,$\dagger$ A. VAN SILFHOUT, $\uparrow$ AND J. ARENDS* \\ * Laboratory for Materia Technica, University of Groningen, Ant. Deusinglaan 1, 9713 AV Groningen, \\ The Netherlands, and †Department of Applied Physics, Twente University of Technology, \\ P.O. Box 217, 7500 AE Enschede, The Netherlands
}

Received November 27, 1984; accepted February 26, 1986

\begin{abstract}
Spreading pressures of water and $n$-propanol on polytetrafluoroethylene (PTFE), polystyrene (PS), polymethylmethacrylate (PMMA), polycarbonate (PC), and glass are determined from ellipsometrically measured adsorption isotherms by graphical integration, yielding for water $9,37,26,33$, and $141 \mathrm{erg} \cdot \mathrm{cm}^{-2}$ on PTFE, PS, PMMA, PC, and glass, respectively, while for $n$-propanol $5,38,26,23$, and $37 \mathrm{erg} \cdot \mathrm{cm}^{-2}$, respectively. The spreading pressures for water as well as $n$-propanol are comparable to values previously obtained from contact angle data with water, water $/ n$-propanol mixtures and $\alpha$-bromonaphthalene using the geometric mean equation. This method yielded spreading pressures of $9,14,30,27$, and $70 \mathrm{erg} \cdot \mathrm{cm}^{-2}$ for PTFE, PS, PMMA, PC, and glass, respectively. The numerical correspondence between the spreading pressures for water and $n$-propanol determined ellipsometrically with the values derived from contact angles indicates the necessity as well as the validity of taking the spreading pressures of water/n-propanol mixtures into account as a constant, if surface free energies of high energy substrata are approximated by contact angle measurements. (c) 1986 Academic Press, Inc.
\end{abstract}

\section{INTRODUCTION}

It is the aim of the present paper to determine spreading pressures of water and $n$-propanol on polytetrafluoroethylene, polystyrene, polymethylmethacrylate, polycarbonate, and glass from ellipsometrically measured adsorption isotherms. Adsorption isotherms can be recorded on flat, nonpowdered samples by ellipsometry, measuring the adsorbed layer thickness as a function of the vapor pressure as has previously been described by Adamson et al. (1-3). Adamson et al. measured isotherms only at relative vapor pressures above 0.5 to 0.6 and employed a potential distortion model together with measured contact angles to derive values for the spreading pressures.

In the present work the use of a potential distortion model is avoided by measuring at relative vapor pressures between 0 and 1.1. Spreading pressures are subsequently calculated by direct graphical integration of the isotherm as

$$
\pi_{\mathrm{e}}=-k_{\mathrm{B}} \cdot T \int_{P_{0}}^{P} \mathrm{\Gamma} \cdot d(\ln p)
$$

where $\pi_{\mathrm{e}}$ is the spreading pressure, $P$ is the vapor pressure, $P_{0}$ is the saturation vapor pressure, $T$ is the temperature, and $\Gamma$ is the number of adsorbed molecules per unit area.

\section{MATERIALS AND METHODS}

Smooth surfaces of PTFE (polytetrafluoroethylene), PS (polystyrene), PMMA (polymethylmethacrylate), PC (polycarbonate), and glass were prepared by grinding and polishing the plate materials, without heating the solids. Prior to each adsorption run samples were kept for $1 \mathrm{~h}$ at $10^{-3}$ Torr.

The water employed in this study was of high purity as required for atomic absorption analysis. $n$-Propanol was obtained from Merck (analytical grade).

Determination of adsorption isotherms. The adsorption experiments were carried out in a double walled, stainless-steel vessel, packed in rockwool. A thermostatically controlled water flow between the two vessel walls provided a temperature control of a few hundredths of a 
degree per degree variation in room temperature.

The vessel was connected with a liquid/saturated vapor reservoir. By varying the temperature of the reservoir the pressure in the vessel could be adjusted. The pressure was measured with a differential pressure transducer 142 PC 01 (Micro Switch).

Adsorption isotherms were determined by means of ellipsometric measurements as described by Tadros et al. (1). The ellipsometric parameters $\Delta$ and $\Psi$ were determined at a wavelength $\lambda=6328 \AA$ with a so-called nulltype ellipsometer.

$\Delta$ and $\Psi$ could be measured with an accuracy of $0.004^{\circ}$ and $0.002^{\circ}$, respectively, but the reproducibility of the experiments was far less. Therefore most adsorption isotherms were recorded several times.

Homogeneous adsorption. In this study the Drude approximation (4), valid if the adsorbed layer thickness is less than the wavelength of the incident light, is used throughout. In the Drude approximation the changes $\delta \Delta$ and $\delta \Psi$ are linearly related to the adsorbed layer thickness:

$$
\begin{aligned}
& \delta \Delta=\zeta \cdot s \\
& \delta \Psi=\eta \cdot s
\end{aligned}
$$

where $\Psi$ and $\eta$ are both complicated functions of the complex refractive indices of the vapor, the adsorbed layer, the solid substrate, the wavelength, and the angle of incidence. Since $\delta \Psi$ was negligible, $\delta \Delta$ was usually the only one measured. The number of adsorbed molecules per unit area can now be calculated directly from

$$
\Gamma=\frac{S}{V_{i}}
$$

in which $V_{i}$ is the molecular volume of the adsorbed molecule.

Heterogeneous adsorption. Heterogeneous adsorption on solid substrata is quite usual in literature and has been encountered in many systems. Theeten (5) as well as Muller and Farmer (6) show that a large increase in $\delta \Delta$ can be accompanied by a smaller increase in adsorbed film thickness than expected on basis of Eqs. [2] and [3] if the film grows heterogeneously (i.e., in patches or islands). In these cases the number of adsorbed molecules can be obtained, employing the assumptions (6) that adsorbed molecules can migrate along the substratum surface to form aggregates (i.e., patches or islands) and that these aggregates are strongly bound to the substratum surface.

In order to calculate $\Gamma$ from measured $\delta \Delta$ values a new parameter $\zeta$ (see Eq. [2]) must be calculated based upon the effective complex refractive index of the heterogeneously adsorbed film.

Assuming that the aggregates form spherical islands having the macroscopic contact angle $\theta$ with the substratum and that they are arranged in a hexagonal array of touching islands (see also Fig. 1), the volume fraction $V_{\mathrm{L}}$ in the adsorbed film can be calculated:

$$
\begin{array}{r}
V_{\mathrm{L}}=\frac{\pi \sqrt{3}}{18} \cdot(1-\cos \theta) \cdot(2+\cos \theta) \cdot \sin \theta \\
\text { for } \theta \geqslant 90^{\circ}
\end{array}
$$
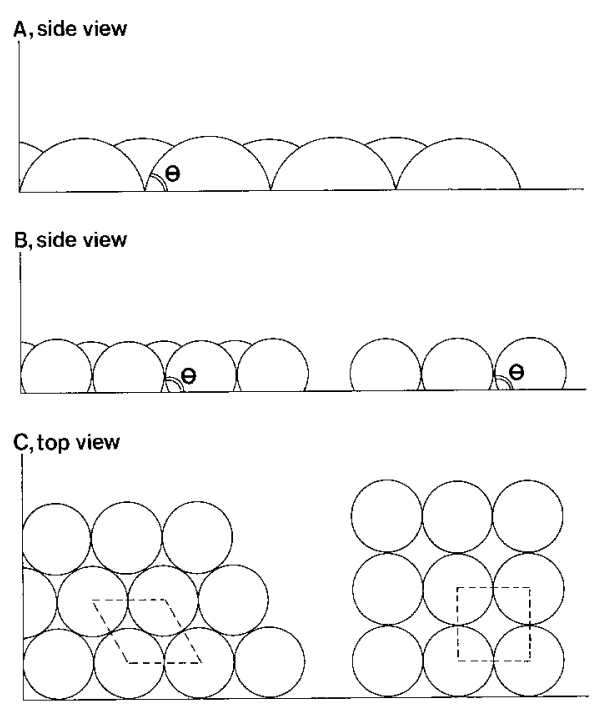

FIG. 1. Schematic illustration of heterogeneities in adsorbed films on solid substrata. (A) Adsorbed microdroplets in an hexagonal array (contact angle $<90^{\circ}$ ). (B) Adsorbed microdroplets in an hexagonal (left) and a rectangular array (right, contact angle $>90^{\circ}$ ). (C) Top view on adsorbed microdroplets arranged in an hexagonal (left) and rectangular array (right). 
$V_{\mathrm{L}}=\frac{\pi \sqrt{3}}{18} \cdot \frac{(1-\cos \theta) \cdot(2+\cos \theta)}{\sin ^{2} \theta}$

$$
\text { for } \theta \leqslant 90^{\circ} \text {. }
$$

Note that $V_{\mathrm{L}}$ is independent of the film thickness.

From $V_{\mathrm{L}}$, the effective complex refractive $\mathbf{n}_{\mathrm{r}}$ of the heterogeneous film, can be calculated using the Maxwell-Garnett formula $(5,6)$

$$
\frac{\mathbf{n}_{\mathrm{r}}-1}{\mathbf{n}_{\mathrm{r}}+1}=\sum_{i} V_{i} \cdot \frac{\mathbf{n}_{i}-1}{\mathbf{n}_{i}+1} .
$$

Subsequently $\mathbf{n}_{\mathbf{r}}$ can be employed to derive a new parameter, $\zeta$, which inserted in Eq. [2] yields a new layer thickness $s$. The number of adsorbed molecules in such an adsorbed film is now given by

$$
\Gamma=V_{\mathrm{L}} \cdot \frac{s}{V_{i}}
$$

\section{RESULTS}

Transforming the measured ellipsometric parameter $\delta \Delta$ into an adsorbed layer thickness provided unexpected difficulties, because for some polymers the $\delta \Delta$ changes could not be explained by pure adsorption only, as shown also by $\delta \Psi$ changes. Desorption experiments gave a clear indication of a small absorption together with the adsorption under study. Furthermore, it was observed that the polymer chains at the surface stretch after vapor pressure increase (7) yielding an unexpectedly slow equilibration of the system, before reversible adsorption of vapor molecules was observed.

In all cases $\Delta$ values of the polymer surface with absorbed material present and with the polymer chains stretched, were taken as a reference for the clean surface. In Fig. 2 some thus obtained adsorption isotherms are shown.

If the homogeneous adsorption model was applied on PTFE and PS, irrealistically thick layers and high values for the spreading pressures were obtained, this indicated that in these cases a heterogeneous adsorption model is valid.

In order to apply the description of the heterogeneous adsorption model, estimates have to be made of the volume fraction adsorbate in the heterogeneous film.

First, spreading pressures derived from graphical integration of the adsorption iso-
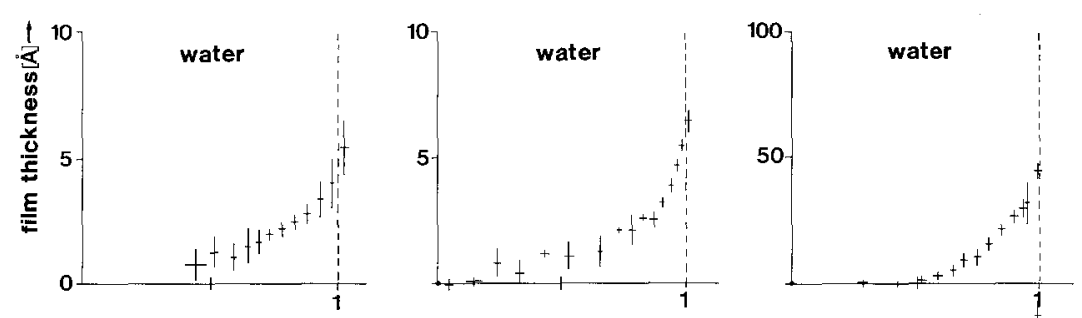

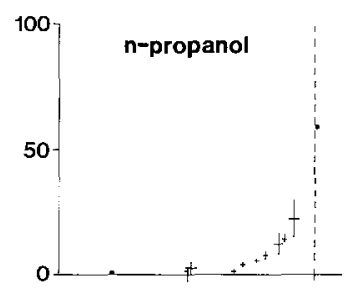

PMMA

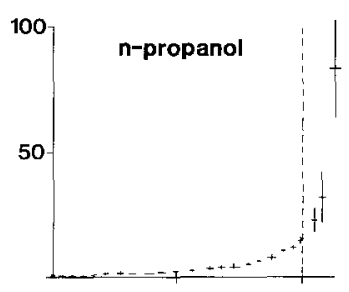

PC

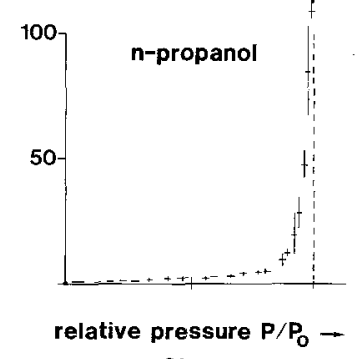

Glass

FIG. 2. Adsorption isotherms for water and n-propanol on PMMA, PC, and glass. The bars denote the standard deviations, arising from three different adsorption runs on various specimens. 
therm were plotted as a function of the volume fraction adsorbate $V_{\mathrm{L}}$ (Fig. 3). It can be seen, that the influence of heterogeneities in the adsorbed film is smaller for $n$-propanol on PTFE, due to the low contact angle and the large difference in refractive index of $n$-propanol and PTFE.

Assuming that the heterogeneously adsorbed film consists of touching, adsorbed spherical islands in an hexagonal array having the macroscopic contact angle as presented in Table II (see also Fig. 1), it's possible to calculate the volume fraction ( $\left.V_{\mathrm{L}}\right)$ from Eqs. [5a] and [5b] yielding values of 0.55 and 0.40 for water and $n$-propanol on PTFE, respectively, and 0.53 and 0.42 for water and $n$-propanol on PS, respectively. Spreading pressures for these systems can subsequently be determined from Fig. 3. It is worth noting, that neither assuming an arrangement of islands in a rectangular (see also Fig. 1) or a random array, nor allowing a small separation between adsorbed islands influences the final values for the spreading pressure appreciably. Spreading pressures for all systems investigated are summarized in Table $\mathrm{I}$.

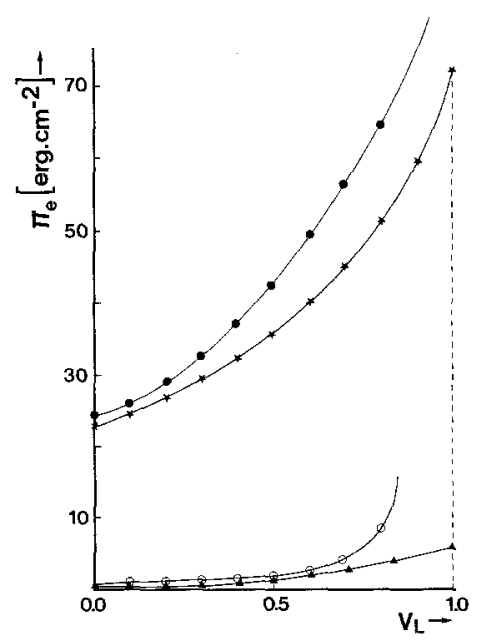

FIG. 3. The spreading pressure obtained by graphical integration as a function of the volume fraction adsorbate in an heterogeneously adsorbed film. (•) $n$-Propanol/PS, (*) water/PS, (O) water/PTFE, (A) $n$-propanol/PTFE.
TABLE I

Spreading Pressures $\left(\mathrm{erg} \cdot \mathrm{cm}^{-2}\right.$ ) Obtained for Water and $n$-Propanol on the Various Solids

\begin{tabular}{lrc}
\hline \multicolumn{1}{c}{ Solid } & Water & $n$-Propanol \\
\hline PTFE & 9 & 5 \\
PS & 37 & 38 \\
PMMA & 26 & 26 \\
PC & 33 & 23 \\
Glass & 141 & 37 \\
\hline
\end{tabular}

\section{DISCUSSION}

Generally, adsorption from a liquid droplet giving rise to spreading pressures will occur if the free energy of the system is thereby reduced. This will occur when the condensed vapor has a surface free energy lower than that of the substratum. Spreading pressures can be determined by several methods (13) such as vapor adsorption isotherms (as done in this study), surface tensions of liquid substrata, and contact angles on solids (as we did previously, see Ref. (8)). A compilation of data for various systems presented by $\mathrm{Wu}$ (13) shows that in general spreading pressures are negligible when the contact angles are large, but can be appreciable when the contact angle approaches zero. Opposite results were reported however by Adamson et al. $(1,2)$, who showed that spreading pressures could become as high as $8.8 \mathrm{erg} \cdot \mathrm{cm}^{-2}$ for water on PTFE, despite the fact that the contact angle is high. This result, obtained by essentially the same ellipsometric technique as employed in this study, compares well with data for water/PTFE presented in this study (see Table I). The high spreading pressures for systems with large contact angles as found by Adamson et al., were denoted by $\mathrm{Wu}(13)$ as anomalies caused by the presence of porosity or hydrophilic sites (introduced during sample preparation) on the solid.

In the present study, absorption of liquid in the solid was accounted for in the evaluation of the ellipsometric data, while during sample preparation no heating was applied in order 
to try to avoid creation of hydrophilic sites. Although this study shows that spreading pressures are highest in systems with the lowest contact angle, spreading pressures were not found to be zero for systems with a high contact angle, in correspondence with data presented by Adamson et al. $(1,2)$.

A method has been published recently (8), which enables the approximation of solid surface free energies as well as of spreading pressures from contact angles. The method is essentially based on least-square fitting of contact angle data from water, water/ $n$-propanol mixtures (up to $40 \mathrm{wt} \% n$-propanol), and $\alpha$-bromonaphthalene to the geometric mean equation $(9,10)$ :

$$
\begin{aligned}
\cos \theta=-1 & +2\left(\gamma_{\mathrm{s}}^{\mathrm{d}} \cdot \gamma_{1}^{\mathrm{d}}\right)^{1 / 2} \cdot \gamma_{1}^{-1} \\
& +2\left(\gamma_{\mathrm{s}}^{\mathrm{p}} \cdot \gamma_{1}^{\mathrm{p}}\right)^{1 / 2} \cdot \gamma_{1}^{-1}-\pi_{\mathrm{e}} \cdot \gamma_{1}^{-1}
\end{aligned}
$$

where: $\gamma_{s}, \gamma_{s}^{\mathrm{d}}$, and $\gamma_{\mathrm{s}}^{\mathrm{p}}$ are the solid surface free energy and its dispersion and polar components, respectively, $\gamma_{1}, \gamma_{1}^{\mathrm{d}}$, and $\gamma_{1}^{\mathrm{p}}$ are the liquid surface free energy and its dispersion and polar components, respectively, and $\pi_{\mathrm{e}}$ denotes the equilibrium spreading pressure.

The major assumptions underlying the method are associated with the spreading pressure term. It was argued that neglect of this term leads to the determination of the solid-vapor interfacial free energy $\gamma_{\mathrm{sv}}$, while taking the spreading pressure into account as a constant, which is assumed to be independent of the $n$-propanol content of the liquid mixtures used, yields the solid-air interfacial free energy $\gamma_{\mathrm{s}}$ and a separate spreading pressure term $\pi_{\mathrm{e}}$.

The spreading pressure of the apolar $\alpha$-bromonaphthalene was assumed to be zero in Eq. [8] (9), enabling direct calculation of $\gamma_{s}^{d}$ from the $\alpha$-bromonaphthalene contact angle.

The authors have employed the above described method to various types of surfaces and noted that the $\gamma_{\mathrm{sv}}$ values obtained were always smaller than 70 to $75 \mathrm{erg} \cdot \mathrm{cm}^{-2}$ and in accordance with literature data. The $\gamma_{\mathrm{s}}$ values obtained however could be as high as 100 to
$140 \mathrm{erg} \cdot \mathrm{cm}^{-2}$ for surfaces as fluorapatite or glass, values which are definitely reasonable compared to literature data $(11,12)$. The difference between $\gamma_{\mathrm{s}}$ and $\gamma_{\mathrm{sv}}$ equaled in all cases the calculated values for the spreading pressure, as it should according to the basic laws of thermodynamics.

Although the contact angle data supported the assumption, that $\pi_{e}$ is independent of the propanol content of the mixtures (correlation coefficients of the data to Eq. [8] were generally higher than 0.97), the exact thermodynamic meaning of the spreading pressure values obtained from the contact angles is not clear, since it is not known whether it belongs to water on the solid substratum, $n$-propanol, or a mixed adsorbed layer of water and $n$-propanol. In this respect, it is interesting to compare values for the spreading pressures of water and $n$-propanol with values determined in the above described way (see Table II).

In Table III spreading pressures obtained from graphical integration are compared with the spreading pressure value obtained from contact angles with water/n-propanol mixtures. In all cases the values obtained are comparable, except for glass, on which $n$-propanol exhibits a far lower spreading pressure than water. The spreading pressure value, obtained from contact angles with water/ $n$-propanol mixtures on glass, however, falls very well be-

\section{TABLE II}

Surface Free Energies and Spreading Pressures (erg $\mathrm{cm}^{-2}$ ) Obtained from Contact Angles with Water, Water/ $n$-Propanol Mixtures and $\alpha$-Bromonaphthalene, and Contact Angles with Water and $n$-Propanol (Degrees) ${ }^{a}$

\begin{tabular}{lrrrrrrr}
\hline \multicolumn{1}{c}{ Solid } & $\gamma_{s}^{\mathbf{d}}$ & $\gamma_{\mathbf{s}}^{\mathbf{p}}$ & \multicolumn{1}{c}{$\gamma_{\mathbf{s}}$} & $\gamma_{\mathbf{s v}}$ & $\pi_{\mathbf{e}}$ & $\boldsymbol{\theta}_{\mathrm{H}_{\mathbf{2}} \mathrm{O}}$ & $\theta_{\boldsymbol{n} \rightarrow \mathrm{prpanol}}$ \\
\hline PTFE & 25 & 0 & 25 & 14 & 11 & 105 & 42 \\
PS & 40 & 4 & 44 & 29 & 14 & 92 & 5 \\
PMMA & 43 & 10 & 53 & 22 & 30 & 76 & 0 \\
PC & 43 & 13 & 56 & 25 & 31 & 86 & 0 \\
Glass & 38 & 100 & 138 & 67 & 71 & 24 & 0 \\
\hline
\end{tabular}

${ }^{a}$ All data apply at $25^{\circ} \mathrm{C}$. 


\section{TABLE III}

$\pi_{\mathrm{e}}\left(\mathrm{erg} \cdot \mathrm{cm}^{-2}\right)$ Data from Water and $n$-Propanol Adsorption Isotherms Obtained by Graphical Integration Compared with the Spreading Pressures from Contact Angles

\begin{tabular}{lccc}
\hline \multicolumn{1}{c}{ Solid } & $\begin{array}{c}n \text {-Propanol } \\
\text { adsorption isotherm }\end{array}$ & $\begin{array}{c}\text { Contact } \\
\text { angles }\end{array}$ & $\begin{array}{c}\text { Water adsorption } \\
\text { isotherm }\end{array}$ \\
\hline PTFE & 5 & 11 & 9 \\
PS & 38 & 14 & 37 \\
PMMA & 26 & 30 & 26 \\
PC & 23 & 31 & 33 \\
Glass & 37 & 71 & 141 \\
\hline
\end{tabular}

tween the values for the pure components. Although the exact thermodynamic meaning of the spreading pressures from contact angles is obscured due to the uncertainties concerning preferential adsorption of $n$-propanol or water, the above comparison strongly indicates that the spreading pressure values from contact angles are quite realistic.

Furthermore, this study shows that although the spreading pressures for water and $n$-propanol are not identical, the variation is small.

Especially when using water $/ n$-propanol mixtures (up to $40 \mathrm{wt} \% n$-propanol) as wetting agents the spreading pressure term in Eq. [8] may therefore be considered as a constant. Since the numerical value of spreading pressures may be appreciable it is not allowed to neglect the influence of the spreading pressure term if solid surface free energies are calculated from contact angles.

APPENDIX: COMPARISON OF VARIOUS METHODS TO DETERMINE SURFACE FREE ENERGIES FROM CONTACT ANGLES

It is interesting to compare various methods to derive solid surface free energies from contact angles and to study the effect of including spreading pressures in the calculations. An advantage of the approach recently outlined (8), and mentioned in this paper, is that it takes spreading pressures into account, a severe disadvantage being the use of liquid mixtures, which has the possibility of showing prefer-

\section{TABLE IV}

Surface Free Energies of the Various Solid Substrata Calculated from the Water and the $\alpha$-Bromonaphthalene Contact Angles Employing the Equation of State $(14,15)$

\begin{tabular}{|c|c|c|c|c|}
\hline Solid & $\begin{array}{c}\theta_{\mathrm{H}_{2} \mathrm{O}} \\
\text { (degrees) }\end{array}$ & $\begin{array}{c}\gamma_{s v}, \mathrm{H}_{2} \mathrm{O} \\
\left(\mathrm{erg} \cdot \mathrm{cm}^{-\mathbf{2}}\right)\end{array}$ & $\begin{array}{c}\theta_{\text {abr }} \\
\text { (degrees) }\end{array}$ & $\begin{array}{c}\gamma_{\mathrm{tv}, \mathrm{arbr}} \\
\left(\mathrm{erg} \cdot \mathrm{cm}^{-2}\right)\end{array}$ \\
\hline PTFE & 105 & 19.2 & 60 & 26.3 \\
\hline PS & 92 & 27.2 & 25 & 40.1 \\
\hline PMMA & 76 & 37.2 & 15 & 42.4 \\
\hline PC & 86 & 31.0 & 15 & 42.4 \\
\hline Glass & 24 & 66.3 & 32 & 37.8 \\
\hline
\end{tabular}

ential adsorption at the various interfaces (3, 13). One of the most commonly applied methods to derive surface free energies is the equation of state approach, this enables the determination of the surface free energy of a solid from contact angle measurements with only one liquid $(14,15)$. Results for the five substrata used in this study based on measured water and $\alpha$-bromonaphthalene contact angles are summarized in Table IV. These surface free energies are in fair agreement with literature data cited by $\mathrm{Wu}$ (13), it is disturbing however that different values for the surface free energy are obtained from the water contact angle and from the $\alpha$-bromonaphthalene contact angle. This may well be related to the fact that water will exhibit different spreading pressures on the substrata than $\alpha$-bromonaphthalene.

\section{TABLE V}

Surface Free Energies of the Various Solid Substrata Calculated from the Water and $\alpha$-Bromonaphthalene Contact Angles Employing the Geometric Mean Equation Neglecting Spreading Pressures

\begin{tabular}{lrrr}
\hline \multicolumn{1}{c}{ Solid } & $\gamma_{\mathrm{wv}}^{\mathrm{d}}$ & \multicolumn{1}{c}{$\gamma_{\mathbf{s v}}^{\mathrm{p}}$} & \multicolumn{1}{c}{$\gamma_{\mathbf{v}}$} \\
\hline PTFE & 24.8 & 0.2 & 25.0 \\
PS & 39.8 & 0.5 & 40.3 \\
PMMA & 40.0 & 4.5 & 44.5 \\
PC & 41.6 & 1.4 & 43.0 \\
Glass & 28.9 & 38.2 & 67.1 \\
\hline
\end{tabular}


A second commonly applied method is the use of Eq. [8] neglecting the spreading pressure term and inserting contact angles with two pure liquids $(10,16)$. Results based on the water and $\alpha$-bromonaphthalene contact angles are summarized in Table $\mathrm{V}$. The data in Table $\mathrm{V}$ appear consistent either with $\gamma_{\mathrm{sv}, \mathrm{H}_{2} \mathrm{O}}$ or $\gamma_{\mathrm{sv}, \alpha-}$ br values presented in Table IV or with literature data cited by $\mathrm{Wu}$ (13).

An elegant approach for deriving surface free energies from Eq. [8] without neglecting spreading pressures and without employing liquid mixtures is offered using the ellipsometrically measured spreading pressures for water. Once $\gamma_{s}^{\mathrm{d}}$ is calculated from Eq. [8] by inserting the $\alpha$-bromonaphthalene contact angle while neglecting the polar interactions and the spreading pressure term, the $\gamma_{\mathrm{s}}^{\mathrm{p}}$ can be calculated from Eq. [8] by inserting the water contact angle, the calculated $\gamma_{\mathrm{s}}^{\mathrm{d}}$, and the ellipsometrically measured spreading pressure for water. Results are summarized in Table VI. The numerical values are generally higher than cited in the literature (13), being comparable for PTFE and being extremely high for glass. Both Table VI as well as Table II show elevated surface free energies $\gamma_{s}$ compared to literature data, though the results in Table VI and Table II are not in complete correspondence with each other. The ex-

\section{TABLE VI}

Surface Free Energies of the Various Solid Substrata Calculated from the Water and the $\alpha$-Bromonaphthalene Contact Angles Employing the Geometric-Mean Equation Neglecting the Spreading Pressure of $\alpha$-Bromonaphthalene while Employing the $\alpha$-Bromonaphthalene Angle but Inserting the Ellipsometrically Measured Spreading Pressures for Water while Employing the Water Angle (see Table I)

\begin{tabular}{lrrr}
\hline \multicolumn{1}{c}{ Solid } & $\gamma_{\mathrm{s}}^{\mathrm{d}}$ & \multicolumn{1}{c}{$\gamma_{\mathrm{s}}^{\mathrm{p}}$} & \multicolumn{1}{c}{$\gamma_{\mathrm{s}}$} \\
\hline PTFE & 25.0 & 1.3 & 26.3 \\
PS & 40.3 & 11.4 & 51.7 \\
PMMA & 42.9 & 15.0 & 57.9 \\
PC & 42.9 & 12.3 & 55.2 \\
Glass & 37.9 & 242.4 & 280.3 \\
\hline
\end{tabular}

tremely high surface free energy $\gamma_{s}$ of glass in Table VI is probably due to the use of the high water spreading pressure, measured on ultraclean glass (in vacuo!).

This comparison of surface free energies derived by various methods, carried out for a limited number of solids, shows that there is a broad correspondence between surface free energy values calculated from contact angles neglecting spreading pressures (Tables IV and V) and literature values from polymer melts, homologuous series, etc. (13). Furthermore this comparison indicates that the inclusion of spreading pressures in the calculation of solid surface free energies from contact angles, may become increasingly important if high energy substrata are to be investigated.

\section{REFERENCES}

1. Tadros, M. E., Hu, P., and Adamson, A. W., J. Colloid Interface Sci. 49, 184 (1974).

2. Hu, P., and Adamson, A. W., J. Colloid Interface Sci. 59, 605 (1977).

3. Adamson, A. W., "Physical Chemistry of Surfaces," 3rd ed. Wiley, New York, 1976.

4. Vašiček, A., "Optics of Thin Films." North-Holland, Amsterdam, 1960.

5. Theeten, J. B., Surf. Sci. 96, 275 (1980).

6. Muller, R. H., and Farmer, J. C., Surf. Sci. 135, 521 (1983).

7. Kip, G. A. M., Busscher, H. J., van Silfhout, A., and Arends, J., Polym. Commun. 26, 215 (1985).

8. Busscher, H. J., van Pelt, A. W. J., Jong, H. P. de, and Arends, J., J. Colloid Interface Sci. 95, 23 (1983).

9. Fowkes, F. M., Ind. Eng. Chem. 56, 40 (1964).

10. Owens, D. K., and Wendt, R. C., J. Appl. Polym. Sci. 13, 1741 (1969).

11. Arends, J., and Jongebloed, W. L., Caries Res. 11, 186 (1977).

12. Aning, M., Welch, D. D., and Royce, B. S. H., Phys. Lett. A 37, 253 (1971).

13. Wu, S., "Polymer Interface and Adhesion," 1st ed. Dekker, New York, 1982.

14. Neumann, A. W., Good, R. J., Hope, S. J., and Sejpal, M., J. Colloid Interface Sci. 49, 291 (1974).

15. Neuman, A. W., Absolom, D. R., Francis, D. W., and Van Oss, C. J., Sep. Purif. Methods 9, 69 (1980).

16. Van Oss, C. J., Good, R. J., and Chandhury, M. K., J. Colloid Interface Sci. 111, 378 (1986). 